\title{
Young adults' images of abstaining and drinking: Prototype dimensions, correlates and assessment methods
}

Journal of Health Psychology 16(3) 410-420

(c) The Author(s) 2010

Reprints and permissions:

sagepub.co.uk/journalsPermission.nav DOI: I0.1 I77/13591053103734/2 hpq.sagepub.com

@SAGE

\section{Friederike Zimmermann \& Monika Sieverding}

University of Heidelberg, Germany

\begin{abstract}
This research investigated contents of actor and abstainer prototypes with regard to young adults' social drinking combining quantitative and qualitative approaches (adjective ratings, $N=300$; open answers, $N=90$ ). Exploratory and confirmatory factor analyses yielded two factors (sociability/hedonism, responsibility) in both prototypes, confirmed by qualitative data. Given the importance for intention and willingness to drink alcohol, interventions should focus on the factor 'sociability/hedonism' of the actor and the abstainer prototype to reduce heavy drinking; addressing 'responsibility' may be ineffective. Participants' evaluations appeared to be less prone to mean tendencies subsequent to open answers compared with adjective scales.
\end{abstract}

\section{Keywords}

alcohol, assessment, evaluation, prototype perception, young adults

The idea of an image associated with a behaviour (e.g. 'toughness' and 'independence' conveyed by smoking cigarettes) as a determinant for behaviour development has been brought into health psychology research about 30 years ago (cf. Leventhal \& Cleary, 1980). Recently, images of abstaining from behaviour (e.g. the 'boring' type of person who does not drink alcohol on a night out) have also been considered. Identification of content dimensions of such actor and abstainer prototypes in relation to young adults' alcohol consumption that may be used in interventions is the main aim of the present work.

One line of research considers such prototypes to be antecedents in behavioural decision making. Depending on the subjective evaluation and perceived similarity, people should intend to distance themselves from a prototype or intend to match it, respectively, in order to enhance or maintain self-consistency and a positive sense of self (Dunning, Perie, \& Story, 1991; Niedenthal, Cantor, \& Kihlstrom, 1985). Another line of research assumes that health-related behaviours are not always deliberate. Instead it suggests that prototypes — particularly with regard to

\footnotetext{
Corresponding author:

Friederike Zimmermann, Psychological Institute, University of Heidelberg, Hauptstraße 47-5I, 69I I7 Heidelberg, Germany. Tel: +49 (0)622I - 54-7297; Fax: +49 (0)622I - 54-7325;
}

Email:friederike.zimmermann@psychologie.uni-heidelberg.de 
(adolescent) health-risk behaviour - also have an influence on behaviour mediated by behavioural willingness (Gerrard, Gibbons, Houlihan, Stock, \& Pomery, 2008). In tempting situations it is one's degree of willingness to be identified as an actor or not to be conceived as an abstainer by others that determines one's own risk behaviour. Altogether, prototypes are investigated regarding their influence on intentions and willingness (e.g. Hyde \& White, 2009; Rivis, Sheeran, \& Armitage, 2006). Moreover, direct effects on behaviour have been found (e.g. Norman, Armitage, \& Quigley, 2007).

Most studies that investigated prototypes of health-related behaviours used a list of predetermined adjectives to derive peoples' evaluations of an image, for example, with regard to the typical smoker (Gibbons \& Eggleston, 1996), the typical drinker (Gerrard et al., 2006), or the typical un/healthy eater (Gerrits, de Ridder, de Wit, \& Kuijer, 2009). Sometimes similarity to the prototype is assessed on a separate item (e.g. Gerrard et al., 2006). Prototype investigations, however, have been limited to such indices of evaluation, similarity, or the combination of both.

If dimensionality of images is identified at all, it is done to obtain indicators for a latent prototype construct, which is interpreted as more or less favourable. This has commonly been done, for example, with regard to children's images of smoking (Gerrard, Gibbons, Stock, Vande Lune, \& Cleveland, 2005) or substance use (Gibbons et al., 2004), and adolescents' drinker and non-drinker prototypes (Gerrard et al., 2002; Ouellette, Gerrard, Gibbons, \& Reis-Bergan, 1999). The content of an image itself has hardly ever been subject to detailed investigation. Hence, although the predictive value of prototypes has been shown by means of similarity and favourability, we know little about the crucial components of potentially heterogeneous images that determine intention, willingness, or behaviour. Altering 'negative' health images or 'positive' risk images by interventions, however, requires an understanding of the relevant factors implied.
Health-psychological research has only recently begun to relate dimensions of prototypes to peoples' behavioural status or intention (Keresztes, Piko, Gibbons, \& Spielberger, 2009; Myklestad \& Rise, 2008; Skalle \& Rise, 2006). Spijkerman, van den Eijnden, Vitale, \& Engels (2004) showed that separate facets of drinker and smoker prototypes predicted adolescents' intention and willingness even in different directions (e.g. 'cool' was positively related to intention and willingness to drink or smoke, whereas 'rebellious' was negatively related to these behavioural antecedents). With regard to young adults' alcohol use, no such analysis exists that may yield more detailed information about which aspects of prototypes should be targeted in interventions.

In addition, it would be valuable to reconsider prototype evaluation. First, derivation of favourability solely from adjective scales appears to be problematic because the direction of evaluation is assumed by the researcher. Whereas evaluation of some items is generally clear-cut (e.g. 'cool' = positive, 'dull' = negative), evaluation of other commonly used items may be ambiguous. For example, 'careless' might be interpreted as neglectful (negative) or as insouciant (positive); young people might construe the (generally positive) attribute 'considerate' as anxious (negative). Second, the adjectives presented might not typify characteristics of a prototype from the individual's perspective. Even if items stem from an elicitation study and represent the most frequently resulting answers, they may not be those the individual conceives as central. This cannot be avoided by the standard introduction of a prototype procedure mentioning that not all actors or abstainers correspond exactly with the typical image, respectively, but many of them share some typical characteristics (cf. Gibbons, Gerrard, \& Boney-McCoy, 1995). The presentation of adjectives may still interfere with people's own conceptions of a prototype. Third, adjective scales of a prototype procedure are usually counterbalanced with respect to evaluation, which may yield artificially balanced responses. 
Adjective scales may thus produce a tendency toward the mean with respect to evaluation.

Few studies asked participants to write down some typical characteristics of a prototype preceding its evaluation, for example, with regard to university students' exercise behaviour (Rivis \& Sheeran, 2003) or binge drinking (Norman et al., 2007). This approach may provide more valid evaluations since people refer to an image in their own words. Usually, in such an open format, specification of three characteristics is requested, which may also be advantageous in yielding more distinct, less artificially balanced results. Thus, it appears to be valuable to compare individuals' subjective evaluations following adjective scales with those following open answers.

The present research focuses on young adults' actor and abstainer images of heightened alcohol use in social contexts. A standard quantitative procedure using adjective scales was employed and was complemented by a qualitative approach that let participants (from a different sample) self-generate characteristics of the prototypes. We aimed to explore a) the dimensionality of the actor and the abstainer prototype, b) the contribution of the factors identified to participants' intention and willingness to drink alcohol during an evening of socializing, and c) the performance of both assessment approaches regarding mean tendencies in evaluations of prototypes.

\section{Method}

\section{Samples and procedure}

Participants in the quantitative prototype approach were $N=300$ young adults (153 male, 147 female; 93\% German) with a mean age of 24.7 years $(S D=3.6)$ who took part in a more extensive prospective study on social-cognitive models explaining young adults' alcohol consumption (Zimmermann \& Sieverding, 2010). Eighty percent of the sample were university students from a range of academic programmes, 13 percent were employed and 7 percent were in training or high school. Ninety-six percent of the sample had attained academic track schools that prepare for university entrance. On average, participants drank several $(>3)$ alcoholic drinks 1.6 times per week $(S D=1.4$, range $=0-7) ; 19$ percent reported 0 days with more than three drinks. Qualitative prototype data were provided by $N=90$ German university students ( 50 male, 40 female) with a mean age of 23.7 years $(S D=$ 3.5 ), also from diverse fields of study. On average, participants drank several $(>3$ ) alcoholic drinks 1.4 times per week $(S D=1.2$, range $=$ 0-4); 20 percent reported 0 days with more than three drinks. Participants in both samples were volunteers recruited on the university campus by experienced research assistants of approximately the same age. The students approached were told that the study was about their beliefs regarding alcohol consumption, which aroused interest in most individuals, and about 80 percent were willing to take part. The study samples and procedures were conducted in accordance with the ethical guidelines of the American Psychological Association (APA); participants were informed about the content of the study preceding data collection, which was not associated with any consequences for the individual, and gave informed consent; confidentiality was guaranteed and contact information was destroyed following data entry.

\section{Measures}

Items used with the quantitative research sample stem from questionnaires of a larger study (Zimmermann \& Sieverding, 2010). Intention was assessed by 'Do you intend to drink several [ $>3$ ] glasses of alcohol tonight?' on a 7-point bipolar scale from 1 (no, in no case) to 7 (yes, in any case). Behavioural willingness was assessed using two scenarios. The participant was asked to imagine to be out with some friends on a Saturday evening, having already consumed quite a number of alcoholic drinks: (1) 'You have the impression that you drank enough. It is about midnight and someone having a birthday is paying for another round', and (2) 'You are entering a flat-rate pub, that is, you can drink as much as you like after having paid the entrance 
fee'. These scenarios were each followed by 'I continue drinking' based on a 7-point bipolar scale from 1 (no, in no case) to 7 (yes, in any case); Cronbach's $\alpha=.86$. A definition built on Gibbons et al., (1995) was given preceding measures of prototype perception. These measures related to the actor prototype (the type of person who consumes several $(>3)$ glasses of alcohol during an evening of socializing) and the abstainer prototype (the type of person who consumes only non-alcoholic drinks during an evening of socializing), respectively. The actor and the abstainer prototype were brought to attention by ratings on 11 7-point semantic differential scales about the general character of such a typical person: sociable (unsociable), willing to take risks(not willing to take risks), open (reserved), reasonable (unreasonable), popular (unpopular), responsible (irresponsible), able to enjoy (unable to enjoy), healthconscious (not health-conscious), easy (uptight), feminine (not feminine), masculine (not masculine). These scales resulted from an elicitation study combined with a review of the literature and from pretesting in additional samples. The order of the actor and the abstainer prototype variables was randomized. Following each prototype's activation, participants gave their subjective evaluation: 'How do you evaluate, all in all, this type of person, who drinks [...] from 0 (extremely unfavourable) to 100 (extremely favourable)?' (cf. Haddock \& Zanna, 1994).

Participants of the qualitative research sample similarly provided evaluations of the prototypes, which had been introduced in the same manner. The only difference was that they were asked to write down three typical characteristics that come to mind to activate the actor and the abstainer prototype, respectively; their order was also randomized in these questionnaires.

Only 2 percent (11 out of 540 characteristics) were missing for the qualitative data. Open answers were first coded into 'positive', 'negative', and 'neutral' regarding their evaluation, and second into categories with regard to the content of the prototype factors - if these were not applicable, into 'other'. Three independent raters coded a random sample of 100 items. Cohen's Kappa ranged from .65 to .76 for agreement in evaluative categories and from .72 to .82 in factor categories. Coding instructions were revised and refined, which led to more reliable agreement (Kappa from .79 to .91 in evaluative categories and from .87 to .97 in factor categories). Coding instructions for the prototype dimensions are available on request from the first author.

\section{Data analyses}

In order to explore the dimensionality of the prototypes and to cross-validate the factors identified, the original quantitative data file was randomly split to conduct exploratory factor analyses (EFA; $N=100)$, which were followed by a confirmatory factor analysis (CFA; $N=$ 200). In EFA, principal axis factoring followed by Promax rotation with Kaiser normalization was employed. Parallel analysis (Horn, 1965) was used to extract all factors whose eigenvalues are greater than those expected from an equivalent random data set using RanEigen (Enzmann, 2003). Items with loadings greater than .45 were considered defining parts of the factors. Fit of the CFA measurement model was inspected using conventional fit indices. To further strengthen the validity of the factors, we controlled whether they were invariant between men and women using a set of multigroup CFA (cf. Meredith \& Teresi, 2006). Correlated uniqueness was allowed (Marsh \& Bailey, 1991) and was constrained to be equal across groups during measurement invariance tests (Vandenberg \& Lance, 2000). Path analysis with latent variables as predictors was conducted to regress intention and willingness to drink alcohol during an evening of socializing on the prototype factors. All analyses involving structural equation modelling were conducted using Mplus (Muthén \& Muthén, 2008). To correct for non-normality, robust maximum likelihood estimation was used.

Favourability-indices were computed for both the quantitative approach (mean of the 
adjective scales) and the qualitative approach (mean of the coded evaluations), and were correlated with participants' subjective evaluations on the $0-100$ scale, respectively. Finally, distributions of subjective evaluations were compared for both approaches and inspected for potential biases, namely in the relative frequency of the neutral mean (50 on the $0-100$ scale).

Less than 1 percent of values were missing for the quantitative variables in both of the data files. Missing data were treated with full information maximum likelihood (FIML) in all analyses involving structural equation modelling. For computation of all other analyses, missing data were estimated using the expectation maximization (EM) algorithm (cf. Graham, 2009).

\section{Results}

\section{Dimensionality of the actor and the abstainer prototype - quantitative data}

Preliminary analyses indicated poor results for the 'feminine' and 'masculine' scales. Since these did not emerge from the preceding elicitation study, but had been supplemented rationally and were mentioned in only two out of the 529 open answers, we considered these items as less relevant for the prototype dimensions and excluded them from the analyses.

Correlation matrices were adequate for EFA as indicated by the Kaiser-Meyer-Olkin measure and the significant Bartlett-test of sphericity for variables of the actor prototype, $\mathrm{KMO}=.78$, $\chi(36)=369, p<.001$, and the abstainer prototype, $\mathrm{KMO}=.81, \chi(36)=419, p<.001$. EFA for adjectives of the actor prototype yielded two factors. The first factor had an eigenvalue of 3.25 and accounted for 36.1 percent of the variance. With regard to content (e.g. 'sociable', 'able to enjoy'), we labelled this factor 'sociability/hedonism'. The second factor had an eigenvalue of 2.46 and accounted for 27.3 percent of the variance. We labelled this factor 'responsibility' (consisting of e.g. 'responsible', 'reasonable'). The same two factors were extracted for adjectives of the abstainer prototype. The first factor had an eigenvalue of 3.70 and accounted for 41.1 percent of the variance. The second factor had an eigenvalue of 2.31 and accounted for 25.6 percent of the variance. Reliability was acceptable to high: actor sociability/hedonism $\alpha=.79$, actor responsibility $\alpha=.81$, abstainer sociability/hedonism $\alpha=$ .84 and abstainer responsibility $\alpha=.84$. The CFA measurement model with all four factors fit the data very well, $\chi^{2}(109, N=200)=125.48$, $p=.134 ; C F I=.98, T L I=.98, R M S E A=.03$, $S R M R=.08$. See Table 1 for factor loadings of the EFA pattern matrices, which exhibited simple structure for both prototypes, and for the standardized factor loadings of the CFA.

In the course of measurement invariance testing based on a set of multigroup models with ascending restrictiveness (Meredith \& Teresi, 2006; Muthén \& Muthén, 1998-2007), none of the restrictions led to significantly worse model fit. The final, most restrictive model, still fit the data well, $\chi^{2}(268, N=200)=318.16, p<0.05 ; C F I=$ $.95, T L I=.95, R M S E A=.04, S R M R=.11$, and not significantly worse compared with the previous model, $\Delta \chi^{2}(18)=15.49, p=.628$, indicating that strict measurement invariance applies to men and women regarding the four factors.

\section{Dimensionality of the actor and the abstainer prototype - qualitative data}

Coding of the open responses into 'sociability/ hedonism', 'responsibility' or 'other' largely supported the factorial solution derived from the quantitative data (see Table 2). With regard to the actor prototype, however, content of responsibility was under-represented compared with that of sociability/hedonism. Residual items still accounted for 27 percent in both the actor and the abstainer prototype and were subject to a more detailed content analysis. Rational sorting yielded the substantial categories of 'aggression', which accounted for 5.7 percent of all open answers, and of 'self-confidence', which accounted for 4.5 percent, leaving a residual category of 16.6 percent.

Plausible differences in frequencies of evaluative terms within the main dimensions of each 


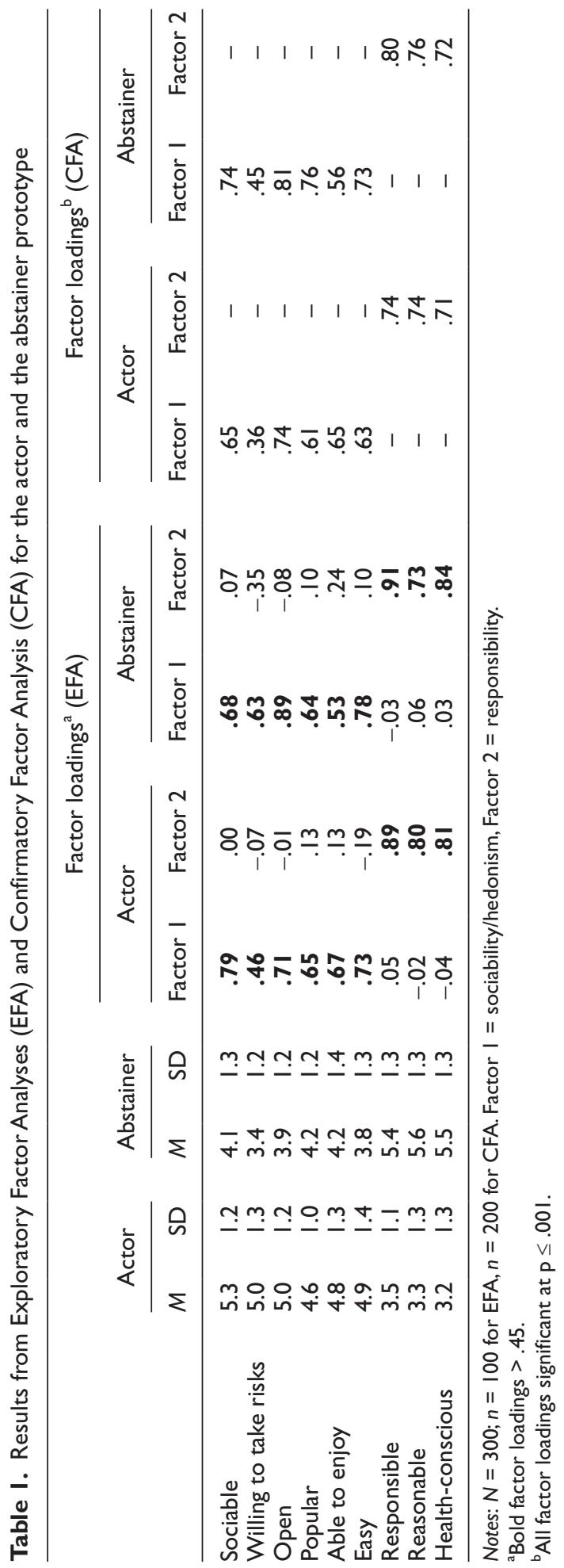


Table 2. Frequencies of open answers in factor categories for the actor and the abstainer prototype and differentiated for evaluative categories

\begin{tabular}{|c|c|c|c|c|c|c|}
\hline & \multicolumn{2}{|c|}{$\begin{array}{l}\text { Sociability/ } \\
\text { Hedonism }\end{array}$} & \multicolumn{2}{|c|}{ Responsibility } & \multicolumn{2}{|c|}{ Other } \\
\hline & $n$ & $\%$ & $n$ & $\%$ & $n$ & $\%$ \\
\hline Actor prototype & 170 & 63.9 & 24 & 9.0 & 72 & 27.1 \\
\hline Positive & 136 & 80.0 & 1 & 4.2 & 10 & 13.9 \\
\hline Negative & 17 & 10.0 & 22 & 91.7 & 52 & 72.2 \\
\hline Neutral & 17 & 10.0 & I & 4.2 & 10 & 13.9 \\
\hline Abstainer prototype & 103 & 39.2 & 90 & 34.2 & 70 & 26.6 \\
\hline Positive & 29 & 28.2 & 75 & 83.3 & 36 & 51.4 \\
\hline Negative & 52 & 50.5 & I & I.I & 18 & 25.7 \\
\hline Neutral & 22 & 21.4 & 14 & 15.6 & 16 & 22.9 \\
\hline
\end{tabular}

Notes: $N=90$.Valid percentages of $n=266$ open answers for the actor prototype and of $n=263$ open answers for the abstainer prototype.

prototype emerged (cf. Table 2). Standardized residuals in cross-tabulation for the actor prototype showed, for example, that positive characteristics were significantly more frequent than negative ones in 'sociability/hedonism'; within 'responsibility', negative characteristics significantly overweighed positive ones, $\chi^{2}(4 ; N=$ $266)=136.90, p<.001$. Regarding the abstainer prototype, negative characteristics were significantly more frequent than positive ones within 'sociability/hedonism'; within 'responsibility', positive characteristics significantly overweighed negative ones, $\chi^{2}(4 ; N=263)=72.24$, $p<.001$. This pattern parallels the prototype dimensions' means, assuming higher favourability with higher values: actor sociability/ hedonism $M=4.94(S D=0.85)$, actor responsibility $M=3.32(S D=1.03)$, abstainer sociability/hedonism $M=3.93(S D=0.93)$, abstainer responsibility $M=5.49(S D=1.13)$.

\section{Regression of intention and willingness on factors of the actor and the abstainer prototype}

Significant differences in the predictive patterns of intention and willingness both as dependent and as independent variables (predicting behaviour) have been found between men and women (Zimmermann \& Sieverding, 2010). Thus, we tested whether different models applied to men and women in this study. The model constrained to be equal across men and women did not fit significantly worse compared with the model with all paths allowed to vary freely, $\Delta \chi^{2}(8)=7.94, p=.440$. Thus, the regression of intention and willingness on the prototype factors was done for the overall sample; model results are given in Table 3 .

The actor prototype dimension 'sociability/ hedonism' played a role both for participants' intention and willingness. The more sociable and hedonistic their image of the typical person consuming several alcoholic drinks, the more they intended to drink several glasses of alcohol during an evening of socializing on the following weekend, and the more they were willing to drink excessively given a tempting social drinking situation. In addition, perceived 'sociability/hedonism' of the abstainer prototype was significantly related to people's willingness. The less sociable and able to enjoy they viewed the typical person drinking only non-alcoholic drinks during an evening of socializing, the more they were willing to drink excessively in a tempting situation. 'Responsibility' was not significantly related to intention or willingness, neither with regard to the actor prototype nor to the abstainer prototype. 
Table 3. Prediction of intention and willingness by latent factors of the prototypes

\begin{tabular}{|c|c|c|c|c|c|c|}
\hline \multirow[t]{2}{*}{ Prototype dimension: } & \multicolumn{3}{|c|}{ Intention } & \multicolumn{3}{|c|}{ Willingness } \\
\hline & $\beta$ & SE & $z$ & $\beta$ & $S E$ & $z$ \\
\hline Actor sociability/hedonism & $.24^{* *}$ & .08 & 2.97 & $.29^{* * * k}$ & .08 & 3.59 \\
\hline Actor responsibility & .10 & .09 & 1.23 & .12 & .09 & 1.28 \\
\hline Abstainer sociability/hedonism & -.08 & .08 & -1.05 & $-.23^{* *}$ & .08 & -2.82 \\
\hline Abstainer responsibility & -.04 & .10 & -0.45 & .04 & .11 & 0.40 \\
\hline$R^{2}$ & .09 & & & .18 & & \\
\hline
\end{tabular}

Notes: $N=300$. Model fit indices: $\chi^{2}(137)=208.87, p<.001 ; C F I=.96, T L I=.94$, RMSEA $=.04$, SRMR $=.07$. $p<.01 ; p<.001$.

\section{Evaluation of the prototypes depending on assessment}

Correlations of derived favourability-indices and subjective evaluation on the $0-100$ scales were all significant at $p<.001$ and were somewhat higher in the qualitative as opposed to the quantitative approach for the actor prototype $(r=.51$ vs. $r=.46)$ and the abstainer prototype $(r=.62$ vs. $r=.56)$ but were not significantly different, respectively.

Distributions of the 0-100 evaluation scale appeared to be more balanced following open answers than adjective scales. Although still overrepresented - without the scales being normally distributed - the relative frequency of the neutral mean on the $0-100$ scale was lower in the qualitative approach compared with the quantitative approach for actor prototype evaluation ( $26.7 \%$ vs. $38.3 \%)$ and abstainer prototype evaluation (18.9\% vs. $37.0 \%)$.

\section{Discussion}

The present study identified dimensions of young adults' actor and abstainer prototypes in relation to alcohol use in social contexts. With regard to our research questions, we found that a) both prototypes consisted of the dimensions 'sociability/hedonism' and 'responsibility', b) exclusively the sociability/hedonism dimensions played a role for participants' intention and willingness, and c) the use of open answers as opposed to adjective scales resulted in a lower tendency toward the mean in evaluations of prototypes. In the following discussion, we consider the conceptual and practical implications of this work.

With regard to content, common dimensions have been identified: 'sociability/hedonism' and 'responsibility' appeared to be appropriate for descriptions of the actor and the abstainer prototype. The factorial pattern was consistent across men and women, which contributes to increased validity, and was largely confirmed by coding of open answers in the qualitative approach. Sorting of residual items yielded additional categories - potential candidates to complement future research on young adults' images of abstaining and drinking, ensuring a more complete picture of the prototypes. Spijkerman et al., (2004) had identified dimensions of adolescents' drinker and smoker prototypes, which appear to be different from those determined in this work. Regarding the prototype of weekly drinking peers, three factors ('well-adjusted', 'cool,' and 'rebellious') had been established from items that had resulted from a literature review and interviews with individuals from the target population, as in our study. The authors found 'cool' to be positively and 'rebellious' to be negatively related to the 14-year-olds' intentions to drink alcohol weekly. These findings are quite different from our own results on young adults. Differences in the drinker image are not surprising, however, since these contents may reflect different themes associated with alcohol use over the developmental course - from identity formation to maintaining the learned standard (cf. the importance of 'sociability/ 
hedonism' for the young adults' intention and willingness to drink alcohol).

The analysis of dimensions is of practical value because it yields more detailed information about which aspects of prototypes should be the target of interventions. As can be concluded according to the regression of intention and willingness, one should focus on 'sociability/ hedonism' of both the actor and the abstainer prototype, whereas addressing the reasonedfocused dimension 'responsibility' may be ineffective. The sociable and hedonistic appearance should be manipulated to present the drinker prototype as more negative or the abstainer prototype as more positive. In contrast, many initiatives aiming to reduce young people's alcohol consumption, such as the current German campaign 'Alcohol? Know your limit. Alcohol destroys more than you think!' (Bundeszentrale für gesundheitliche Aufklärung, n.d.), primarily addresses 'responsibility'. Materials from that campaign show fun-having young people drinking in their usual social drinking situations. The texts that are superimposed over their heads indicate their impending consequences, and the descriptive text on the webpage concludes that the take-home message is: 'The responsible handling of alcohol is essential.' Although this is completely right, focusing on the risks of alcohol and appealing to people's reason - while displaying the sociable and hedonistic qualities of drinking - may not change their behaviour.

Predictors of intention and willingness were equal for men and women in this study, and prototypes may, in principle, influence behaviour through both of these variables. A prospective study on young adults, however, has shown that intention was a significant predictor among men and women, whereas willingness predicted only men's alcohol consumption (Zimmermann \& Sieverding, 2010). Therefore, it has to be kept in mind that predictors of willingness may have higher relevance in young men with regard to the amount of alcohol consumed: 'sociability/hedonism' of both prototypes may be crucial only for men's social-reaction path to behaviour. The relevance of the typical person drinking only nonalcoholic drinks being viewed as unsociable and unable to enjoy for increased willingness, is consistent with the finding that men high in willingness drank to excess particularly when they evaluated the abstainer image as negative (Zimmermann \& Sieverding, 2010). The image of the typical person drinking several glasses of alcohol as sociable, however, appears to be important for men's and women's intentional behaviour of having some alcoholic drinks during an evening of socializing.

Independent from the resulting prototype dimensions, it needs to be clarified whether participants' subjective evaluation should be obtained following adjective scales (cf. eg. Gibbons et al., 1995) or open-ended questions (cf. eg. Rivis \& Sheeran, 2003). Our results show limitations in the use of adjective scales in a prototype approach when assessing evaluation, given the high percentage of those who chose 50 on the $0-100$ scale. This tendency toward the mean may indicate that a considerable number of participants evaluated the prototypes with reluctance or driven by (counter-balanced) adjective scales instead of responding openly. Evaluations must be provided as frankly as possible, however, in order to draw conclusions about their impact on intentions, willingness and behaviour. Thus, evaluations following open-ended questions should be preferred.

\section{Limitations and recommendations for future research}

Our findings are limited by several limitations. First, prototype perception was assessed in a within-subjects-design with possible distortions due to contrasting the actor prototype against the abstainer prototype. Although we diminished this problem by randomizing the order of prototype variables, it is recommended that future research uses a between-subjects design instead. Second, the single-item rating for intention makes it impossible to determine its reliability, and future studies should make an effort to use a multiple-item measure. Third, the overly high tendency toward the mean in evaluation following ratings on adjective scales compared with open-ended questions might 
alternatively be explained by the items 'masculine' and 'feminine', which were only seldom given in participants' open answers or in the preceding elicitation study. Particularly, it might have been these two items that have caused people to provide such a huge number of neutral evaluations. Correlations between these two items and the evaluation scale were, however, in the same range as for the other adjectives. Moreover, it is unclear why the content of just these two items - in contrast to the others - would be related to tendentious evaluation judgments. Fourth, the advantage of subjective evaluations subsequent to open answers compared with adjective scales for the prediction of intention and willingness or behaviour has not been shown in this work and is a task that remains for future research.

Although important questions remain, we would like to provide the following recommendations. First, the results highlight more generally the importance of investigations into dimensions of health-related prototypes and their role for behavioural antecedents to enhance our understanding of the essential contents of interventions. Second, if evaluation of a prototype is of interest, we would recommend to explicitly ask for participants' subjective evaluations (e.g., on a 0-100 scale), since they do not have to match those evaluations previously assumed: they were not very highly correlated with evaluations derived from adjective scales or from coded open answers. Scholars of the prototype/ willingness approach proposed evaluating the prototype, after a short time of contemplation, on a list of adjectives or followed by an open-ended evaluation (cf. Gibbons, Gerrard, \& Lane, 2003). We recommend avoiding adjective scales and making use of open answers in order to reduce tendencies toward the mean in prototype evaluations.

\section{Acknowledgement}

We thank Sabine C. Koch for her helpful comments on an earlier draft of this manuscript.

\section{Competing Interests}

None declared.

\section{References}

Bundeszentrale für gesundheitliche Aufklärung (n.d.). Alkohol? Kenn dein Limit. Alkohol macht mehr kaputt, als du denkst! http://www.kenndein-limit.info (accessed 11 March 2010)

Dunning, D., Perie, M., \& Story, A. L. (1991). Self-serving prototypes of social categories. Journal of Personality and Social Psychology, 61, 957-968.

Enzmann, D. (2003). RanEigen, Version 2.0 [Computer Software]. Hamburg: Universität Hamburg.

Gerrard, M., Gibbons, F. X., Brody, G. H., Murry, V. M., Cleveland, M. J., \& Wills, T. A. (2006). A theory-based dual-focus alcohol intervention for preadolescents: The strong african american families program. Psychology of Addictive Behaviors, 20, 185-195.

Gerrard, M., Gibbons, F. X., Houlihan, A. E., Stock, M. L., \& Pomery, E. A. (2008). A dual-process approach to health risk decision making: The prototype willingness model. Developmental Review, 28, 29-61.

Gerrard, M., Gibbons, F. X., Reis-Bergan, M., Trudeau, L., Vande Lune, L. S., \& Buunk, B. (2002). Inhibitory effects of drinker and nondrinker prototypes on adolescent alcohol consumption. Health Psychology, 21, 601-609.

Gerrard, M., Gibbons, F. X., Stock, M. L., Vande Lune, L. S., \& Cleveland, M. J. (2005). Images of smokers and willingness to smoke among african american pre-adolescents: An application of the prototype/willingness model of adolescent health risk behavior to smoking initiation. Journal of Pediatric Psychology, 30, 305-318.

Gerrits, J. H., de Ridder, D. T. D., de Wit, J. B. F., \& Kuijer, R. G. (2009). Cool and independent or foolish and undisciplined? Adolescents' prototypes of (un)healthy eaters and their association with eating behaviour. Appetite, 53, 407-413.

Gibbons, F. X., \& Eggleston, T. J. (1996). Smoker networks and the 'typical smoker': A prospective analysis of smoking cessation. Health Psychology, $15,469-477$.

Gibbons, F. X., Gerrard, M., \& Boney-McCoy, S. (1995). Prototype perception predicts (lack of) pregnancy prevention. Personality and Social Psychology Bulletin, 21, 85-93. 
Gibbons, F. X., Gerrard, M., \& Lane, D. J. (2003). A social-reaction model of adolescent health risk. In J. M. Suls \& K. A. Wallston (Eds.), Social psychological foundations of health and illness (pp. 107-136). Oxford, England: Backwell.

Gibbons, F. X., Gerrard, M., Vande Lune, L. S., Wills, T. A., Brody, G., \& Conger, R. D. (2004). Context and cognitions: Environmental risk, social influence, and adolescent substance use. Personality and Social Psychology Bulletin, 30, 1048-1061.

Graham, J. W. (2009). Missing data analysis: Making it work in the real world. Annual Review of Psychology, 60, 549-576.

Haddock, G., \& Zanna, M. P. (1994). Preferring 'housewives' to 'feminists': Categorization and the favorability of attitudes toward women. Psychology of Women Quarterly, 18, 25-52.

Horn, J. L. (1965). A rationale and test for the number of factors in factor analysis. Psychometrika, 30, 179-185.

Hyde, M. K., \& White, K. M. (2009). Similarity not favourability: The role of donor prototypes in predicting willingness to donate organs while living. Journal of Health Psychology, 14, 888-898.

Keresztes, N., Piko, B. F., Gibbons, F. X., \& Spielberger, C. D. (2009). Do high- and lowactive adolescents have different prototypes of physically active peers? The Psychological Record, 59, 39-52.

Leventhal, H., \& Cleary, P. D. (1980). The smoking problem: A review of the research and theory in behavioral risk modification. Psychological Bulletin, 88, 370-405.

Marsh, H. W., \& Bailey, M. (1991). Confirmatory factor analyses of multitrait-multimethod data: A comparison of alternative models. Applied Psychological Measurement, 15, 47-70.

Meredith, W., \& Teresi, J. A. (2006). An essay on measurement and factorial invariance. Medical Care, 44, S69-S77.

Muthén, L. K., \& Muthén, B. O. (1998-2007). Mplus user's guide, 5 edn. Los Angeles, CA: Muthén \& Muthén.

Muthén, L. K., \& Muthén, B. O. (2008). Mplus version 5.2 [Computer Software].

Myklestad, I., \& Rise, J. (2008). Predicting intentions to perform protective sexual behaviours among Norwegian adolescents. Sex Education, 8, 107-124.

Niedenthal, P. M., Cantor, N., \& Kihlstrom, J. F. (1985). Prototype matching: A strategy for social decision making. Journal of Personality and Social Psychology, 48, 575-584.

Norman, P., Armitage, C. J., \& Quigley, C. (2007). The theory of planned behavior and binge drinking: Assessing the impact of binge drinker prototypes. Addictive Behaviors, 32, 1753-1768.

Ouellette, J. A., Gerrard, M., Gibbons, F. X., \& Reis-Bergan, M. (1999). Parents, peers, and prototypes: Antecedents of adolescent alcohol expectancies, alcohol consumption, and alcoholrelated life problems in rural youth. Psychology of Addictive Behaviors, 13, 183-197.

Rivis, A., \& Sheeran, P. (2003). Social influences and the theory of planned behaviour: Evidence for a direct relationship between prototypes and young people's exercise behaviour. Psychology \& Health, 18, 567-583.

Rivis, A., Sheeran, P., \& Armitage, C. J. (2006). Augmenting the theory of planned behaviour with the prototype/willingness model: Predictive validity of actor versus abstainer prototypes for adolescents' health-protective and health-risk intentions. British Journal of Health Psychology, 11, 483-500.

Skalle, S., \& Rise, J. (2006). The relationship between smoker and nonsmoker prototypes and smoking status among 14-year-old Norwegians. Addictive Behaviors, 31, 57-68.

Spijkerman, R., van den Eijnden, R. J. J. M., Vitale, S., \& Engels, R. C. M. E. (2004). Explaining adolescents' smoking and drinking behavior: The concept of smoker and drinker prototypes in relation to variables of the theory of planned behavior. Addictive Behaviors, 29, 1615-1622.

Vandenberg, R. J., \& Lance, C. E. (2000). A review and synthesis of the measurement invariance literature: Suggestions, practices, and recommendations for organizational research. Organizational Research Methods, 3, 4-69.

Zimmermann, F., \& Sieverding, M. (2010). Young adults' social drinking as explained by an augmented theory of planned behaviour: The roles of prototypes, willingness, and gender. British Journal of Health Psychology, 15, 561-581. 